\title{
An ICT Platform for Building Analytics
}

\author{
K.I. Katsigarakis \& G. D. Kontes \\ Department of Production Engineering and Management, Technical University of Crete, Greece
}

J. Rojicek

Honeywell Labs, Prague, Czech Republic

C. Valmaseda \& J.L. Hernandez

Department of Energy, Fundación CARTIF, Valladolid, Spain

D.V. Rovas

Department of Production Engineering and Management, Technical University of Crete, Greece \& System Integration Group, Fraunhofer Institute for Building Physics, Nuernberg

ABSTRACT: Significant research effort is underway towards designing intelligent BEMS, adapted to the properties of each building at hand and to the microclimatic conditions of the building location, complemented by a collection of assessment services. On the other hand, the adoption of such solutions by the market is still hampered by the necessity of engineering experts to take over system installation on buildings. Thus, optimizing the energetic performance of buildings is not only about defining intelligent services, but also providing an ICT platform able to host and manage a collection of such analytics. In the proposed approach, such a platform is developed to facilitate the provision of building analytics services. Open source technologies and service-oriented architectures establish the openness, transparency and interoperability characteristics of the platform, while development of generic analytics allows applicability to a large portion of the existing buildings stock in Europe and world-wide.

\section{INTRODUCTION}

Buildings account for a large part of the total energy consumption, with the greater amount of this energy utilized to ensure comfortable interiors. This implies that efficient Building Energy Management Systems (BEMS) design can lead to significant energy savings.

Significant research effort is underway towards designing intelligent BEMS, adapted to the properties of each building at hand and to the microclimatic conditions of the building location (Constantin et al., 2013; Kontes et al., 2014; Oldewurtel et al., 2012; Sturzenegger et al., 2013; Zavala, 2013), but although all these novel approaches have exhibited significant results, they facilitate a divergent list of functional requirements, such as building models of varying complexity or specialized optimization software.

On the other hand, smart or intelligent building does not necessarily mean only well controlled building, but also fault-free building, where faults are related to all equipment operated in the building. In view of this, today's Building Management Systems (BMS) play an essential role in managing buildings by providing some monitoring and control capability for multiple sub-systems including HVAC, electrical systems, fire systems, security systems and others, but yet the importance of the assessment part is still underestimated.

Although building optimization and assessment services emerge as a viable solution to the problem of designing intelligent BEMS, the adoption of such solutions by the market is still hampered by the necessity of engineering experts to take over system installation on commercial buildings. Thus, optimized building operation is not only about defining intelligent optimization and assessment services (or analytics), but also providing an ICT platform able to host and manage in a transparent manner a collection of such analytics, possibly contributed by different stakeholders.

In this direction, within BaaS FP7 project, such a platform is developed, along with a set of analytics for building optimization. The utilization of open source technologies and service-oriented architectures (Valmaseda et al., 2013) establishes the openness, transparency and interoperability characteristics of the platform, while proper selection and development of specific analytics allows applicability to a large portion of the existing buildings stock in Europe and world-wide.

In the present paper, the functionality of the supported groups of analytics is presented, as well as a detailed analysis on the platform components that are responsible for hosting and managing these services. In Section 2 a general overview of the architecture of the platform is provided; Section 3 presents the different types of analytics and their hosting environment, Section 4 provides a detailed description of the data access and communication mechanisms that support the analytics functionality, 
while Section 5 contains some concluding and future work remarks.

\section{THE BAAS PLATFORM}

BaaS ICT platform conceptualizes a "plug-and-play" infrastructure, able to deploy and manage all necessary components towards such solution. In this direction, it is understood that for the typologies of all buildings considered, the topic of energy management and fault detection is an inherently complex one, with this complexity stemming from the pure nature of the application domain. Each building is different: these differences range from the construction and geometrical characteristics, the building installed energy systems, the building energy management system and its configuration, prevailing weather conditions, occupancy and usage patterns to name but a few. In that sense, a simple one-sizefits-all type of solution is not possible as it will have very limited applicability and little to no replicability potential.

To alleviate these shortcomings we take the stance within BaaS that a modular component-based approach can be much more suitable for addressing a significant number of use cases and problems. In that sense, we identify a number of component blocks that provide specific analytics (e.g. thermal comfort calculation in a zone, detection of a "stuck" sensor, temperature setpoint regulation based on forecasts, etc.) that can be used towards developing a tailored strategy to each deployment scenario. These functional blocks are referred to within BaaS as APO services. The term APO collectively refers to continuously recurring tasks during building operation: (A)ssessment of the current building state; (P)rediction of the effects that various decisions will have on Key Performance Indicators (KPIs); and, (O)ptimization of performance as measured through relevant KPIs. The successful demonstration of BaaS hinges upon two conditions:

1. The availability of a platform that is capable of hosting these services and supporting their data requirements;

2. The development of reusable components - APO modules - that can be applicable in a large number of usage scenarios.

Regarding the platform, within the BaaS architectural design detailed in (Cahill et al., 2012; Valmaseda et al., 2013) and illustrated in Figure 1, a three layer architecture is envisaged:

- The Data Layer (DL) is the layer which provides and stores all raw data. Here, there are several data sources in the plant (i.e. the physical building) which provide data about the state of the building and its equipment and resources, e.g. the BMS, a local weather station, or an ac- cess control system. Moreover, there are the two main databases of the BaaS system, holding static geometric data (Building Information Management server (Eastman et al., 2011)) and dynamic fact data, configuration parameters, and APO outputs (Data Warehouse). Finally, all external data sources, e.g. external weather services, are considered part of the Data Layer;

- The APO Service Layer (APO-SL) provides the reasoning and analytics services. The services hosted in the APO-SL make extensive use of the data provided by the DL.

- The Communication Logic Layer (CLL) acts as an abstraction layer to facilitate communication between the Data Layer and the APO Service Layer. On the one hand, the CLL glues the DL and the APO-SL together but, on the other hand, hides them from each other. That is, access from the APO-SL to the DL is greatly simplified using the CLL while at the same time the APOSL does not need to know any details of the data layer but only uses a single, well-defined interfaces which is provided by the CLL. By means of this interface, all communication requirements of the APO-SL with the DL can be satisfied.

It is important to emphasize here that these functionally disjoint layers operate independently and, as a result, they can be hosted in different geographical locations.

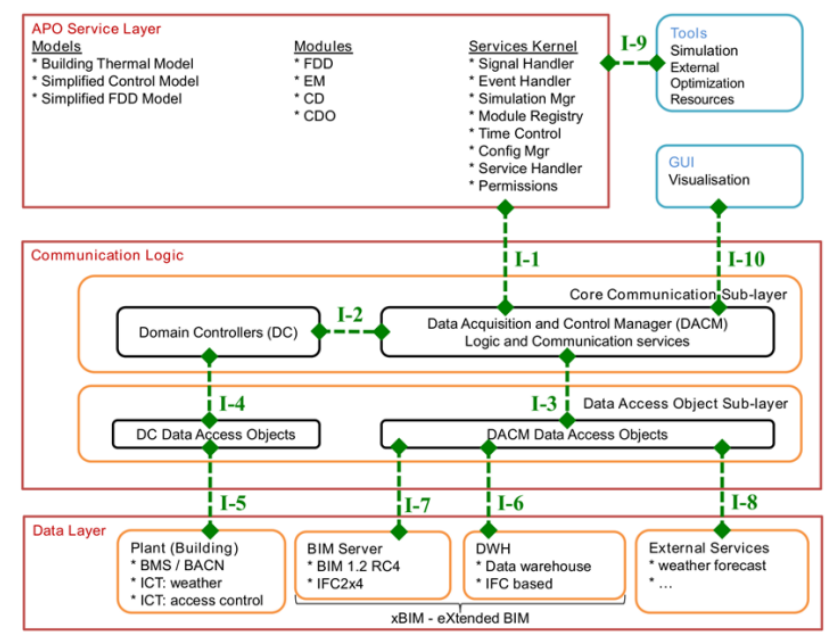

Figure 1: BaaS high-level architecture

\section{THE APO SERVICE LAYER}

Functionally, the APO services layer is intended to host the necessary algorithms to analyze the collected in-building data, to manage interactions of processes and generate control strategies for effective energy management. Specifically, the APO services provide core intelligence, building/facility assessment and monitoring, prediction and optimization services, utilizing information made available by the 
data layer services. Such functionality can be delivered in three hierarchical layers:

- Field layer comprises primarily sensors (temperature, humidity, interior air quality, occupancy detectors, etc.) and actuators (valves, light switches, etc.);

- Automation layer consists of controllers whose logical functions may range from the single input single output (SISO) closed-loop control at the base level, to plant-level control and supervisory control or optimization at the upper level;

- Management system layer provides capabilities for monitoring and centralized management of the building. At this layer BMS can provide various advisory services for the people involved in daily operation, such as service technicians, engineers, or facility managers. Frequently these services may take advantage of powerful cloudbased analytics, which complement the functionality delivered by BMS locally on given site.

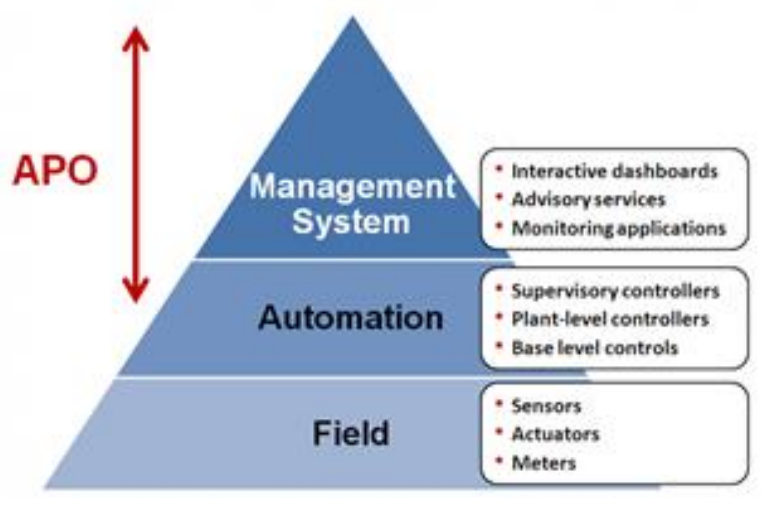

Figure 2: Functional layers of Building Management Systems

In this layer, what is collectively denoted as services should be understood as functional components implemented as a collection of software modules. These modules are either developed during BaaS project or can be provided by interested stakeholders to implement analytics (fault detection and diagnostics, control design, etc.). From a business perspective these modules can be part of the business intelligence and solutions portfolio provided as a service to building owners and occupants.

In the remaining of this Section a brief description on the envisioned properties of each of the defined functional groups of services (assessment, optimization and prediction) is provided. For more details on the defined list of services, we refer the interested reader in (Rovas et al., 2014).

\subsection{Assessment}

The objective of assessment APOs is to monitor the performance of various building systems, detecting inefficiencies or faults, or processing the collected data to create new information or insights into the building operation without actually modifying building behavior; found issues are reported to the facility manager to take relevant actions, thus assisting towards optimal maintenance planning (Zhang and Gao, 2012). Many of assessment tasks face the challenge of scalability, from both a technical and business perspective. Human resources are scarce and expensive, so automation is needed in the analytics to support human analysts. From a business perspective, analytics must be highly repeatable at low cost, as the profit margin on data services is often much lower than the profit margin on controls hardware and software solutions - all these aspects lay a solid foundation for assessment APOs need. Assessment analytics can be divided into 2 subtasks:

- Performance assessment. The goal of such services is just to assess the performance of the system and describe it by chosen characteristics called Key Performance Indicators (KPIs), containing services which provide analytics to monitor equipment performance at various building hierarchy levels (from the building envelope to the individual building equipment) and to identify critical levels for effective operation in order to take measures for respective maintenance;

- Fault detection and diagnostics. Unlike performance assessment, the goal is to detect abnormal situation possibly with its root cause. The possible faults fall under two categories:

- Hard faults- hardware faults(typically abrupt changes or equipment malfunctions);

- Soft faults- performance degradation monitoring (typically faults with slow dynamics).

\subsection{Optimization}

In this category supervisory control strategies are mainly considered, since lower-level tasks, like valve controls and pump operation, are tasked to the building's PLCs that are better suited for supporting the real-time requirements of these low-level functionalities.

In view of this, model-based control design strategies (Kontes et al., 2014) are considered that optimize and automatically tune the system parameters so that better performance - with respect to energetic and comfort criteria - is achieved, supported by data-driven approaches (Macek et al., 2013) in case a proper model of the building is not available, thus providing a rich portfolio of supported scenarios.

For the model-based optimization, a thermal model of the building is assumed available, and is utilized to predict cost function values (e.g. total energy consumption or operational cost) and (visual, thermal, etc.) comfort constraints using available weather and occupancy forecasts, while for the data- 
driven approach, historical data from the building relevant to the optimization task, are assumed to be available.

\subsection{Prediction}

Improving operational performance utilizing both assessment and optimization analytics requires better planning and utilization of resources. Prediction of future behavior can be a valuable tool towards achieving this improved performance. Thus, a service that is able to predict future states of the building is essential for the optimization process, while the availability of models for the entire building and HVAC sub-systems can provide valuable input to various assessment analytics. Here, a wide variety of approaches for defining such a prediction module are supported, facilitating different properties. An extensive study of the literature reveals a natural, high-level categorization of these predictors (or models) in three categories with increasing complexity:

- Data-driven models, generated by aggregation techniques over existing building data (Macek et al., 2013);

- First-principles models, constructed using coupled differential equations describing the physical phenomena occurring in the building (Lilis et al., 2013; Oldewurtel et al., 2012; Zavala et al., 2010);

- Detailed thermal simulation models, encapsulating a large variety of HVAC and energy systems, like EnergyPlus (Crawley et al., 2001), TRNSYS (Klein et al., 1976) or Modelica (Wetter, 2009).

\subsection{The APO Kernel}

The APO kernel is tasked to manage the installation, configuration and complex interplay of the available APO services, as well as the data exchange of the APO services Layer to the other layers of the BaaS platform. A high-level schematic of the APO Kernel is shown in Figure 3, where the core of the kernel is shown in blue, comprising of the following components:

- Building Connector Layer (Signal Abstraction);

- Data Management Layer (Schedule Abstraction);

- Module Manager Layer (Process Abstraction).

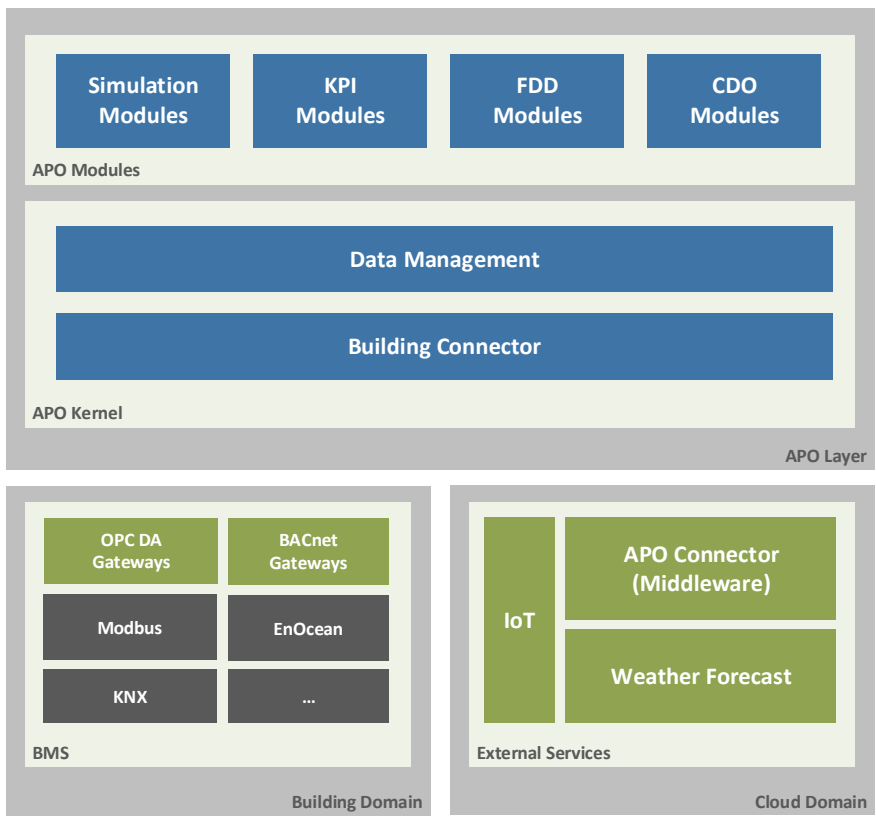

Figure 3: Integration with the other domains

More precicely, the "Building Connector" layer, shown in Figure 4, includes:

- The "Building Connector" embedded services (configuration manager, event producer, unit converted, etc);

- Drivers for BMS Gateways (OPC DA, BACnet, etc.) as reference services to the Signal Handler;

- APIs for the web-enabled devices (Internet of Things devices, weather stations, etc.) as reference interfaces to the Signal Handler;

- APIs for the external services as reference interfaces to the Signal Handler;

- The "APO Connector" bundle for the communication with the Middleware as reference service to the Signal Handler.

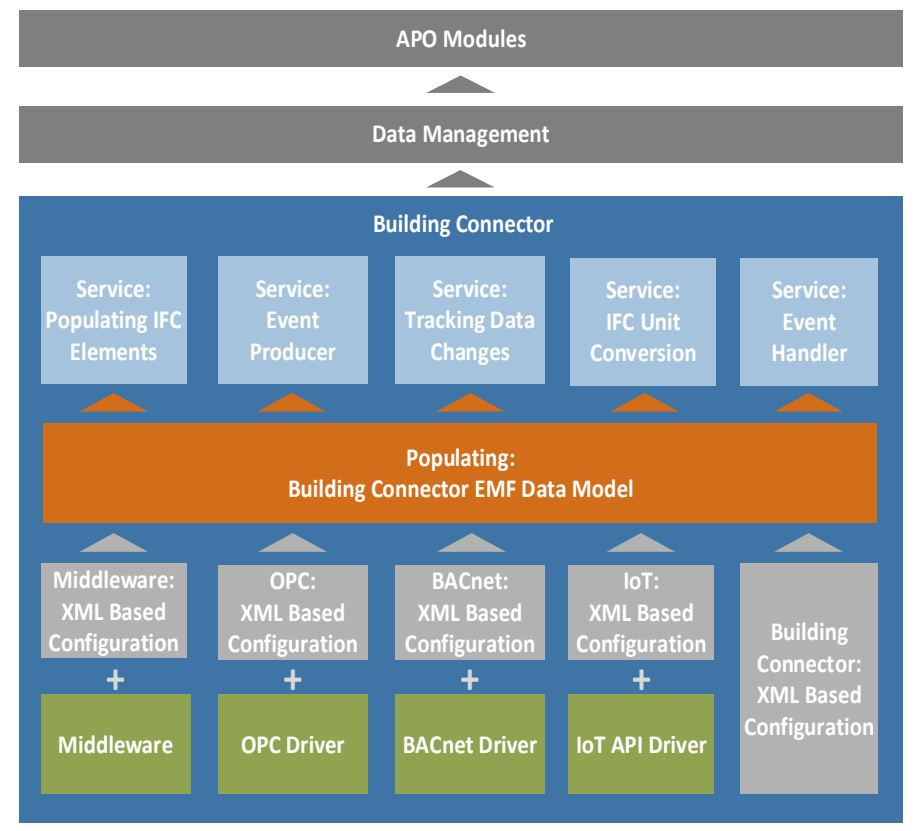

Figure 4: Building Connector Layer 
Each software component or API has a configuration XML file based on a validated XSD schema of the Signal Handler. The initialization of the services is based on an abstracted data model (EMF). The "Signal Handler" bundle includes the following services:

- Service for tracking the signal actual data for changes. The service uses configuration parameters per signal or per signals group;

- Classes for unit conversions based on the IFC data model and for the construction of IFC elements like IfcSensor or IfcActuator with associations to the IfcMeasureWithUnit for scalar data or to the IfcIrregularTimeSeries for vector data;

- Event producer service in order to inform the subscribers on specific topics when the signal data changes;

- Event handler service for the incoming events from the sender "APO Modules" like IFC resource elements (e.g. IfcMeasureWithUnit, IfcIrregularTimeSeries) with new data for setting or storing in the external data domains (e.g. building domain, cloud domain).

The "Data Management" layer shown in Figure 5 includes:

- DB/DW Connector: Implements low-level connectivity for historical data access and provides standard interfaces for data access. It is based on an abstracted data model (EMF) for the initialization of the embedded services. It's simple to develop new extensions of the databases by keeping the same configuration schema of the core services;

- Datapoint Manager: Implements the configuration services of the schedule abstraction and keeps on memory the well-known intervals for each datapoint object. More than one datapoints can be available for a given schedule (predicted, simulated, historical, etc.) and a preference over data from specific origin may apply to the configuration parameters;

- Data Logger: Implements a very simple logic "when an event arrives record it" or recording the last event according to a fixed schedule;

- Schedule Data Access: Implements the interface which is depended on the "Datapoint Manager" and "DW Connector" bundles and provides homogeneous data access interfaces to the APO Modules.

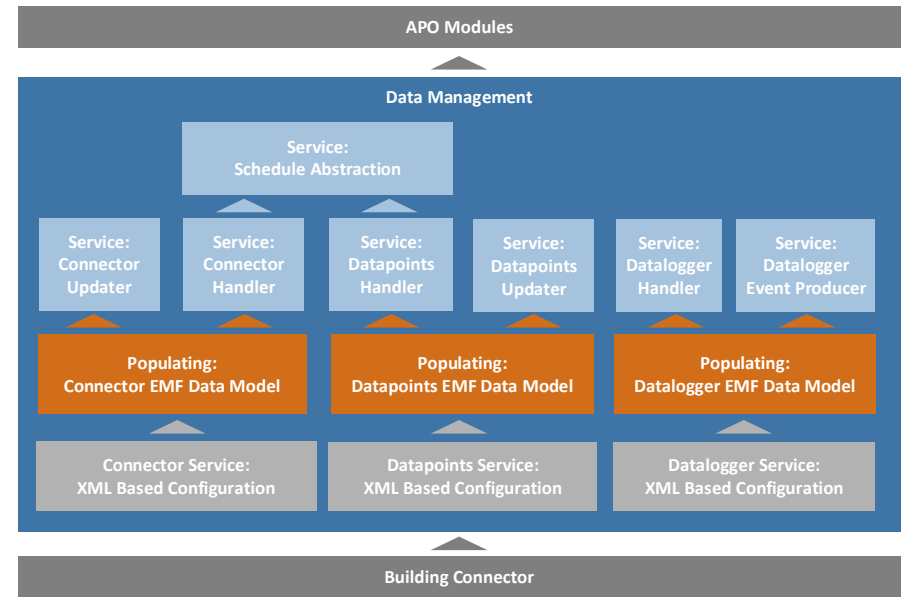

Figure 5: The data management layer

The "APO Modules" layer shown in Figure 6 includes:

- Actor System. Implements an actor based system in order to simplify the construction and the execution phase of the concurrent components (modules). The service uses an embedded MoML parser in order to load the configuration and the wiring parameters;

- Event Handler. Implements the event admin interface in order to handle events from internal or external sources (e.g. virtual signals) or alarms (e.g. for triggering modules);

- Classes for unit conversions based on the IFC data model and for populating IFC resource elements like IfcMeasureWithUnit for scalar data and IfcIrregularTimeSeries for vector data;

- Event Producer, which informs the subscribers such as "APO Modules" or "DW Connector" with new data (e.g. optimized vector data, predicted vector data).

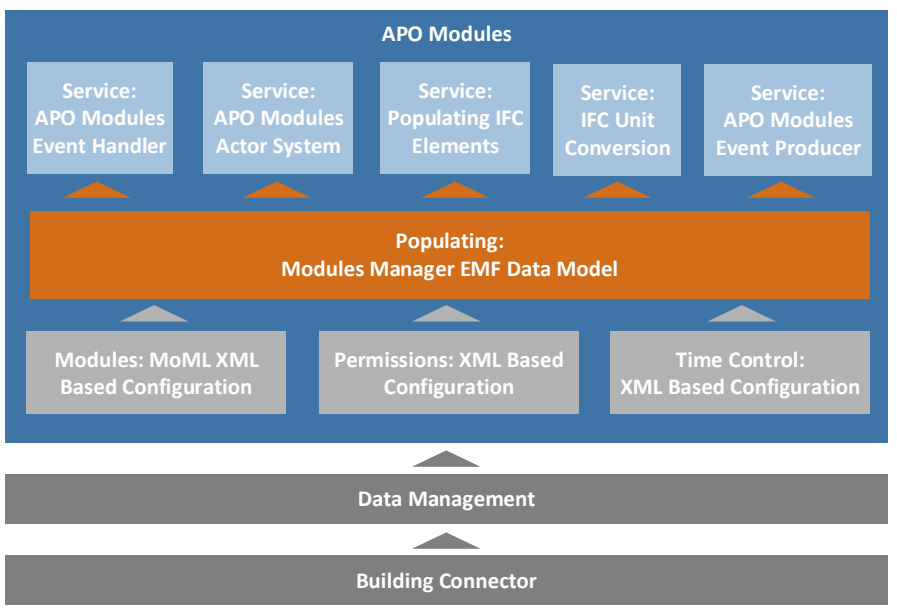

Figure 6: The APO modules layer

Finally, Figure 7 illustrates the interaction of the APO kernel with the implemented APO modules. The "Building Connector" layer includes a tracking service, where for each signal, one IFC object 
(IfcMeasureWithUnit, IfcRegularTimeSeries, etc.) is populated according to the data type (scalar, vector etc.) each time the value changes. The new value is wrapped to an IFC object and converted from the BMS units to the default unit template of the platform. As next step the OSGi "EventAdmin" service produces an asynchronous event with the basic parameters (signal, timestamp and the populated IFC object).

The "Data Management" layer, includes the Data Logger which holds the updated IFC object and according to the configuration type (event driven or fixed interval) decides when to record the value. The "Data Management" layer also maintains some meta-data information for each data-point concerning the data origin, the data type and the units.

An "APO Module" sends a request to the "Data Management" layer in order to execute the hosting logic. Additionally to the ID and the definition interval of the data, the request may include some preferences for the data (data origin, template for unit conversion, interpolation parameters, etc.). As result of the execution, the "APO Module" populates an IFC object with the "optimized data" and produces an asynchronous event to the "Building Connector" for the related signal.

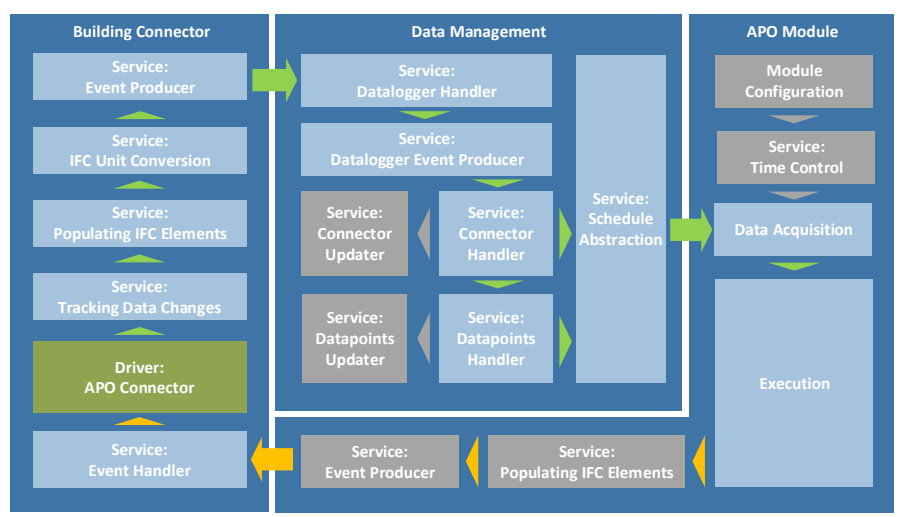

Figure 7: APO Kernel - APO modules interaction

\section{DATA ACCESS AND COMMUNICATION}

As described above, APO services cannot be defined as standalone modules, since their functionality hinges on external to the APO layer components, with the most critical being the Data Layer, which serves the data requirements of the analytics. The Data Layer in BaaS system facilitates the following four main components/data sources:

- The Building Management System (BMS), which is a core component to any building control solution. BMSs act as information gateways for communicating information from/to building sensing and actuation modalities;
- The Data Warehouse (DW), a tool which supports the management and processing of granular bulk data. A great benefit of using a DW is the possibility of post-processing information so that relevant to the end-user KPIs can be computed. Users and analytics services can directly retrieve and utilize the pre-computed values. In an effort to ensure interoperability between all BaaS components, the BaaS data warehouse schema has been developed in conformance with the ISO 16739:2013 standard (IFC4) (Cahill et al., 2012; ISO, 2013);

- The BIM server, which hosts all IFC related data and supports queries to the data model for retrieving building-related information on demand. The desire for openness has led to the use of the TNO BIM Server (http://bimserver.org/), an open source server. The code has been properly adapted within BaaS in order to provide basic support to the IFC4 schema;

- External Data Services (ED), for retrieving building-related data from sources external to the BMS. Such data may e.g. include: weather forecasts, occupant density data (or occupant profiles from security systems) or occupancy forecasts (from occupancy scheduling systems).

The final piece of the puzzle is the Communication Logic Layer, acting as a middleware, linking the Data Layer and the APO Layer in a bi-directional manner. The CLL is an abstract and interoperable layer which communicates the different data sources in a transparent way (Hernandez et al., 2013; Martin et al., 2013) and consists of the Domain Controller(s) (DC) and the Data Acquisition and Control Manager (DACM) as illustrated in Figure 8.

The Domain Controller runs on the site of the asset at hand. It provides low-level access to the physical asset, e.g. by connecting to the BMS for data retrieval or actuation triggering. In case of very large or distributed assets (e.g. large industrial plants or operating sites of a company spread over a large geographical area), more than one DC can be utilized, as shown in Figure 8. This implies that multiple DCs can be managed by a single DACM to monitor and control a single asset or that multiple assets can be controlled simultaneously by a single DACM or a combination of both.

The DACM is linked to the $\mathrm{DC}(\mathrm{s})$ and provides high-level functionality, e.g. connectors to components outside the CL, such as APO services or external cloud-based data sources (weather data, etc.). In the same way that one DACM can control multiple DCs, a plenitude of APO services can be connected to a DACM (Valmaseda et al, 2013). 


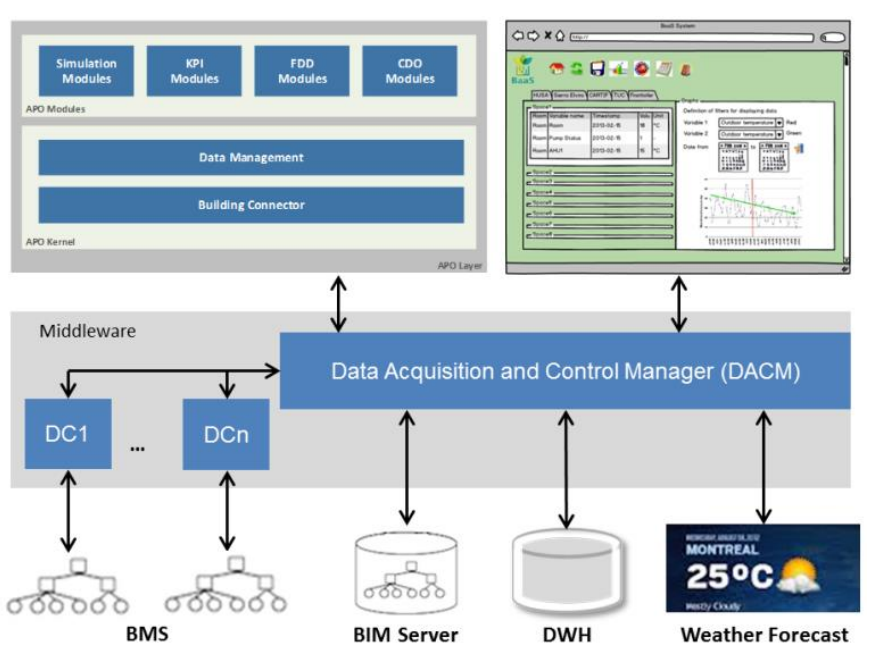

Figure 8: Distributed BaaS platform

The access to the Data Layer is carried out through well-established interfaces (Hernandez et al, 2013), implemented by proper connectors that translate the raw data into IFC4 compliant information, thus ensuring interoperability throughout BaaS platform.

The low-level communication mechanism is based on events provided by OSGi framework (OSGi Alliance, http://www.osgi.org). These events are associated to properties that share the useful information among entities. Thus, for example, the CLL is able to maintain a historical record of the data measurements logged periodically from the BMSs and weather forecast services. In addition, the CLL offers interoperability with the different instances deployed of the resources without the knowledge of the deployment scheme from the upper layers (i.e. APO-SL).

Last but not least, a graphical user interface provides the access to the visualization and configuration functionalities.

\section{CONCLUSIONS}

In the present work, an ICT platform for hosting and managing building performance optimization analytics is presented. The three-layer architecture of the platform is presented, along with an analysis on the functional properties of the APO layer hosting the modular services, as well as the data access and communication components supporting these analytics. In addition, the properties of each group of APO services (assessment, optimization and prediction) are also presented.

As development of each of the three layers has been completed within BaaS FP7 project, next steps include the successful combined operation of all components of the platform, as well as application to all BaaS demonstration buildings.

\section{AKNOWLEDGEMENTS}

The research leading to these results has been partially funded by the European Commission FP7ICT-2011-6, ICT Systems for Energy Efficiency under contract \#288409 (BaaS).

\section{REFERENCES}

Cahill, B., Menzel, K., Floeck, M., Schmidt, M., Schulke, A., Etinski, M., Hernandez, J.L., Martin, S., Valmaseda, C., 2012. High-Level Architecture, Interfaces Definitions, Data Models Extension Description. BaaS Project Deliverable.

Constantin, A., Fütterer, J., Streblow, R., Müller, D., Kontes, G.D., Rovas, D.V., 2013. Simulation assisted implementation of a model based control parameter fine-tuning methodology for a nonresidential building with a complex energy system, in: Proceedings of BS 2013: 13th Conference of the International Building Performance Simulation Association. pp. 1869-1876.

Crawley, D.B., Lawrie, L.K., Winkelmann, F.C., Buhl, W.F., Huang, Y.J., Pedersen, C.O., Strand, R.K., Liesen, R.J., Fisher, D.E., Witte, M.J., Glazer, J., 2001. EnergyPlus: creating a new-generation building energy simulation program. Energy and Buildings 33, 319-331. doi:10.1016/S03787788(00)00114-6

Eastman, C., Teicholz, P., Sacks, R., Liston, K., 2011. BIM handbook: A guide to building information modeling for owners, managers, designers, engineers and contractors. Wiley. com.

Hernandez, J.L., Floeck, M., Schmidt, M., Mo, K., Rojicek, Jiri, Katsigarakis, K.I., Rovas, D.V., 2013. Interfaces to External ICT systems, Interfaces Definition, Mo, K., Rojicek, J., Rovas, D., Kontes, G., Katsigarakis, K. BAAS Deliverable 3.3.

International Organization for Standarization, 2013. ISO16739:2013: Industry Foundation Classes (IFC) for data sharing in the construction and facility management industries. Geneva, Switzerland: International Organization for Standardization.

Klein, S.A., Beckman, W.A., Duffie, J.A., 1976. TRNSYS - A TRANSIENT SIMULATION PROGRAM., in: ASHRAE Transactions. pp. 623-633.

Kontes, G.D., Valmaseda, C., Giannakis, G.I., Katsigarakis, K.I., Rovas, D.V., 2014. Intelligent BEMS design using detailed thermal simulation models and surrogate- based stochastic optimization,. Journal of Process Control. doi:http://dx.doi.org/10.1016/j.jprocont.2014.04.003

Lilis, G.N., Sklivaniotis, K., Giannakis, G.I., Rovas, D.V., 2013. SRC: A SYSTEMIC APPROACH TO BUILDING THERMAL SIMULATION. Presented at the Building Simulation Conference, BuildSim 2013, Chamberry, France, pp. 3199-3192.

Macek, K., Rojicek, J., Kontes, G., Rovas, D.V., 2013. Black-Box Optimization for Buildings and Its Enhancement by Advanced Communication Infrastructure. ADCAIJ: Advances in Distributed Computing and Artificial Intelligence Journal 1, 53-64. 
Martin, S., Hernandez, J.L., Floeck, M., Schmidt, M., Mo, K., 2013. Development guide. BAAS Deliverable.

Oldewurtel, F., Parisio, A., Jones, C.N., Gyalistras, D., Gwerder, M., Stauch, V., Lehmann, B., Morari, M., 2012. Use of model predictive control and weather forecasts for energy efficient building climate control. Energy and Buildings 45, 15-27.

Sturzenegger, D., Gyalistras, D., Gwerder, M., Sagerschnig, C., Morari, M., Smith, R.S., 2013. Model Predictive Control of a Swiss office building, in: 11th REHVA World Congress Clima.

Valmaseda, C., Garcia Fuentes, M.A., Hernandez, J.L., Katsigarakis, K.I., Kontes, G.D., Rovas, D.V., 2013. An Event-driven SOA-based Platform for Energyefficiency Applications in Buildings, in: Proceedings of 30th International Conference on Applications of IT in AEC Industry (CIB W78 2013).

Wetter, M., 2009. Modelica-based modelling and simulation to support research and development in building energy and control systems. Journal of Building Performance Simulation 2, 143-161.

Zavala, V.M., 2013. Real-Time Optimization Strategies for Building Systems. Ind. Eng. Chem. Res. 52, 3137-3150. doi:10.1021/ie3008727

Zavala, V.M., Wang, J., Leyffer, S., Constantinescu, E.M., Anitescu, M., Conzelmann, G., 2010. Proactive energy management for next-generation building systems, in: Proceedings of 4th International Conference of IBPSA-USA.

Zhang, X., Gao, H., 2012. Determining an optimal maintenance period for infrastructure systems. Computer-Aided Civil and Infrastructure Engineering 27, 543-554. 